\title{
Fast Obstacle Detection by Variable Step of 3D Laser Scanning for Robot Navigation on Unknown Planet
}

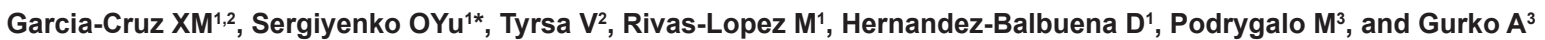

${ }^{1}$ LOAM, UABC, Mexicali, Baja California, México

2ITMexicali, Mexicali, Baja California, México

${ }^{3} \mathrm{KNAHU}$, Kharkov, Ukraine

\begin{abstract}
In the exploration of another planet by mobile robot the slow 3D scanning caused by small step can be a problem. It can be increased by combined scanning step for faster search of $\mathrm{n}$ obstacles in unknown surrounding. This is of keynote importance in automatic robot navigation, especially on the surface of another planet. To maintain a reasonable speed robot must to detect dangerous obstacles as soon as possible, and then calculate a safe trajectory in real time. So, the scanning with variable speed and precise digital mapping only for selected spatial sectors is under consideration here. Wide range of simulations in MATLAB of several scenes with variable $n$ obstacles, scanning it with angle value since $0.6^{\circ}$ up to $15^{\circ}$ was provided aiming to detect such angular values still permitting to get the most information about obstacles without undesired time loss. Three of such angles were obtained in simulation and then rectified by Levenberg-Marquradt Algorithm application and were applied to micro electro transmission design for practical realization of variable combined step scanning on our previously known laser scanner.
\end{abstract}

Keywords: Planet exploration; Mobile robot; TVS; Scanning step; NN; Micro Electro-Mechanic Transmission

\section{Introduction}

The exploration on other planet usually is special task for robot navigation vision, optical scanning with laser is attractive to use over others approaches. When the navigation needs: direct information acquisition, low noise, acceptable accuracy with low uncertainty, ability to work in complete darkness, laser scanning is the one option. Optical sensors give reliability and data stability, can see a real trend in the noncontact sensors based in optical technologies [1]. Contactless acquire a large number of points. Furthermore, laser sensors currently on the market do not have the accuracy of the dynamic contact sensors. But, this accuracy depends on different parameters: surfaces color, texture, materials properties.

Recent research [2] tells diverse optical methodologies have been implemented in tracking systems, could for example make do into [2,3]. In MacFarlane et al [4] was demonstrated two-dimensional $x-y$ laser scanning, using a computer controlled, and rotating reflective cube. Advance the simple/durable optic scanning method which is able to give still more open angle-of-view than any known techniques, except the omni-directional vision (fish-eye lens) [5]. Application depth map by an efficient epipolar plane analysis method $[6,7]$ was analyzed on [8] for omni-directional stereo vision. In application on another planet additionally is growing the possibility to detect something absolutely unknown on Earth, so it is impossible navigation based on analysis of camera images [9]. The laser scanner developed in [10] does not provide $3 \mathrm{D}$ shape information of the detected obstacles because of the absence of enough computational power for 3D shape reconstruction. The avoidance strategy proposed in [10] is to avoid obstacles regardless of their shape. A good review of 3D model acquisition techniques can be found in $[11,12]$. The most crucial point in this case is a time of scanning. Obstacle must be detected in a shortest possible time, but the precise coordinate's measurements is not necessary for all obstacle shape, but only for its edge nearest to the robot's desired safe trajectory (see $[9,13,14]$ ). This paper, modeling sundry scenes with geometrical obstacles, for lay down simulation tool to find optimal angles which will be combined-step scanning, purpose of design based on stepper-motors in laser positioning system, designing a micro electromechanical transmission for that job.

\section{Problem statement}

Our previous research [9,13-19], TVS we allow 3D Cartesian coordinates measurements for obstacles inside scene during navigation. TVS can give a digital map of the obstacles within the Field-of-View (FOV). The current TVS use constant scanning pitch which is not optimally efficient for robot navigation. The objective in unknown scenes is the scanning with combined scanning step, which will minimize scanning time.

We introduce basic principle of dynamic triangulation in [9,13-19], TVS works under dynamic triangulation theory in order to obtain 3D coordinates of Sij points on objects, as shown in Figure 1 and Figure 2, laser beam is projected by Positioning Laser (PL) into obstacle surface, reflecting back is captured by revolving sensor called Scanning Aperture (SA) [20].

TVS has registered two dynamic angles $\mathrm{Ci}$ and $\mathrm{Bj}$ [14].This system is able to track any point in front of the mobile robot calculating the instant triangle with two known angles and precisely known fixed distance between PL and SA.

In order to calculate any coordinate in Cartesian plane was used sine laws on dynamics triangulation by the following equations [17].

*Corresponding author: Oleg Sergiyenko, LOAM, UABC, Mexicali, Baja California México; Tel: 52-686-566-4150; E-mail: srgnk@iing.mxl.uabc.mx

Received April 20, 2013; Accepted August 27, 2013; Published August 29, 2013

Citation: Garcia-Cruz XM, Sergiyenko OY, Tyrsa V, Rivas-Lopez M, HernandezBalbuena D, et al. (2013) Fast Obstacle Detection by Variable Step of 3D Lase Scanning for Robot Navigation on Unknown Planet. J Appl Mech Eng 2: 124 doi:10.4172/2168-9873.1000124

Copyright: (c) 2013 Garcia-Cruz XM, et al. This is an open-access article distributed under the terms of the Creative Commons Attribution License, which permits unrestricted use, distribution, and reproduction in any medium, provided the original author and source are credited. 
Citation: Garcia-Cruz XM, Sergiyenko OY, Tyrsa V, Rivas-Lopez M, Hernandez-Balbuena D, et al. (2013) Fast Obstacle Detection by Variable Step of 3D Laser Scanning for Robot Navigation on Unknown Planet. J Appl Mech Eng 2: 124. doi:10.4172/2168-9873.1000124

Page 2 of 6

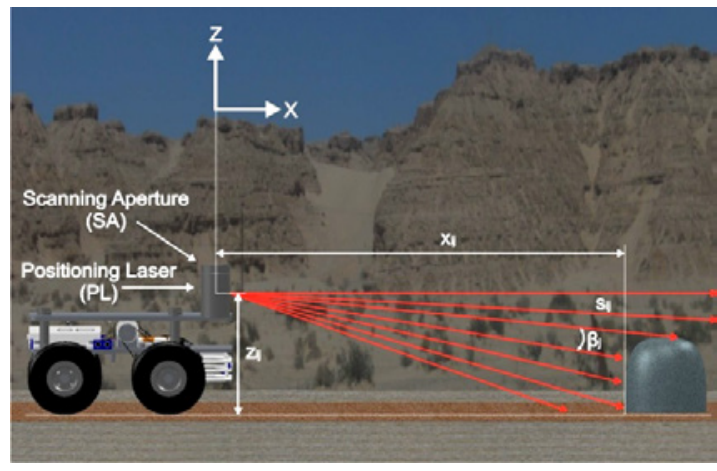

Figure 1: TVS Side view [7].

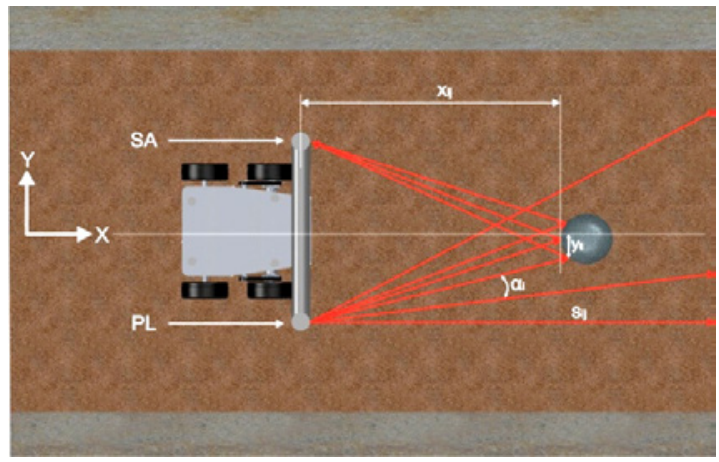

Figure 2: TVS Top view [7]

$$
x_{i j}=a \frac{\sin B_{i j} \cdot \sin C_{i j} \cdot \cos \sum_{j=1}^{j} \beta_{j}}{\sin \left[180^{\circ}-\left(B_{i j}+C_{i j}\right)\right]},
$$

$y_{i j}=a\left(\frac{1}{2}-\frac{\sin B_{i j} \cdot \cos C_{i j}}{\sin \left[180^{\circ}-\left(B_{i j}+C_{i j}\right)\right]}\right)$ at $\mathrm{Bij} \leq 90^{\circ}$,

$$
y_{i j}=-a\left(\frac{1}{2}+\frac{\sin B_{i j} \cdot \cos C_{i j}}{\sin \left[180^{\circ}-\left(B_{i j}+C_{i j}\right)\right]}\right) \text { at } \mathrm{Bij} \geq 90^{\circ}
$$

$$
z_{i j}=a \cdot \frac{\sin B_{i j} \cdot \sin C_{i j} \cdot \sin \sum_{j=1}^{j} \beta_{j}}{\sin \left[180^{\circ}-\left(B_{i j}+C_{i j}\right)\right]}
$$

Application in PL of TVS Micro-Electromechanical Transmission (MET) with combined scanning step. This is the most permissive of possible ways in resolution constraints improvement [11]. Some of them can be ignored under the simple criterion that robot cannot path there. Information about obstacles can be reflected by the number of detected 3D points on the obstacle. In Figure 3 is shown the proposal MET.

\section{How simulation was?}

The next frame shown a scene (see Figure 4) shown: step angle between two consecutive positions of laser ray, points which the system detected, are the locations were laser ray is reflected to scanning aperture (Intersection Point Of Simulated Laser Ray With The Obstacle), and figures within the scene are simulations of obstacles. TVS FOV: beginning at 10 degrees and finish at 170 degrees. Within present scene (sizes: $30 \mathrm{~m}$ width and $15 \mathrm{~m}$ depth).

To simulate laser ray, which unfortunately due to MATLAB'S nature must to have discretization for finite time of calculations, the following model based on Euler's identity was used [21]:

$$
A e^{i \theta}=A \cos \theta+i A \sin \theta
$$

Where $\theta$ is current step angle, $A$ is the length of ray

For simulated obstacles were used next lines equations $Y_{r 1}, Y_{r 2}, Y_{r 3}$ in order to draw a triangle:

$$
\begin{aligned}
& Y_{r 1}=\frac{\left(y_{1-} y_{2}\right)}{\left(x_{1-} x_{2}\right)}\left(x-x_{1}\right)+y_{1} \\
& Y_{r 2}=\frac{\left(y_{2}-y_{3}\right)}{\left(x_{2-} x_{3}\right)}\left(x_{2}-x_{1}\right)+y_{2} \\
& Y_{r 3}=\frac{\left(y_{1}-y_{3}\right)}{\left(x_{1-} x_{3}\right)}\left(x_{3}-x_{1}\right)+y_{3}
\end{aligned}
$$

Where $x_{1}, x_{2}, x_{3}$ and $y_{1}, y_{2}, y_{3}$ are Cartesian coordinates of the triangle vertexes.

For simulated circles were used arbitrary radio $(r)$ and center $(C)$, and discretization of the circle $(D C)$ in grade [20]:

$$
\Delta \alpha=\frac{(D C \times \pi)}{180^{\circ}}
$$

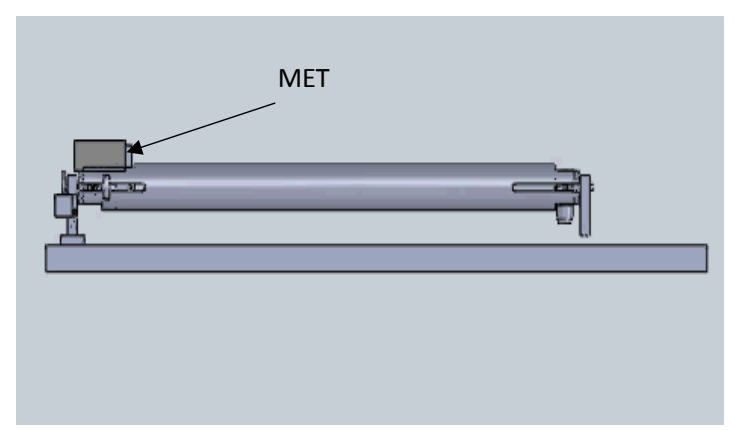

Figure 3: TVS with the gear box proposal [21].

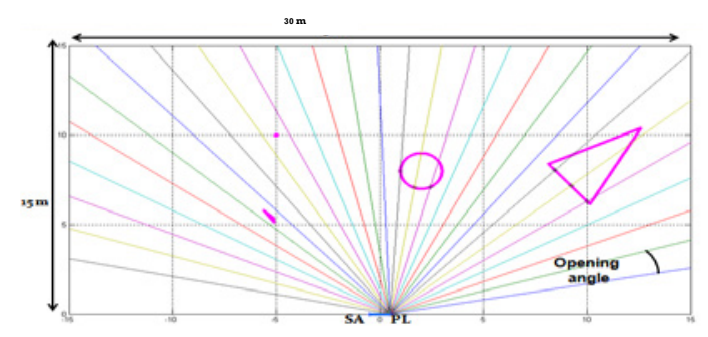

Figure 4: Scene [14]. 
Where $\alpha$ are increments of $\Delta \alpha$ and $\Delta \alpha$ is the increment in the circle obstacle discretization.

$$
\text { Circle }_{n}=C+r e^{j \times \alpha}
$$

Where Circle $_{n}$ is the obstacle drawn for $n$ circles.

\section{Parameters and obtained frames analysis}

Simulation was 101 different scenes, which varied next parameters [22]:

1. Opening angle ( 0.6 degrees to 15 degrees with $\Delta \theta=0.1455^{\circ}$ for each step)

2. Number of obstacles (1 to 13 )

3. Obstacle position (Random)

4. Obstacle dimensions (Random)

Figure 5 show us some scenes of simulation, each scene was scanned with 100 different step angles and varying parameters thus obtaining 10,100 frames to analyze, the notes were:

1. Smaller obstacles and near positioned, were difficult to detected, need angle around 0.6 to 0.7455 .

2. On Figure $5 \mathrm{a}$ with 8 small obstacles and opening angles $15^{\circ}$ and $14.8545^{\circ}$ detected one obstacle in minor time.
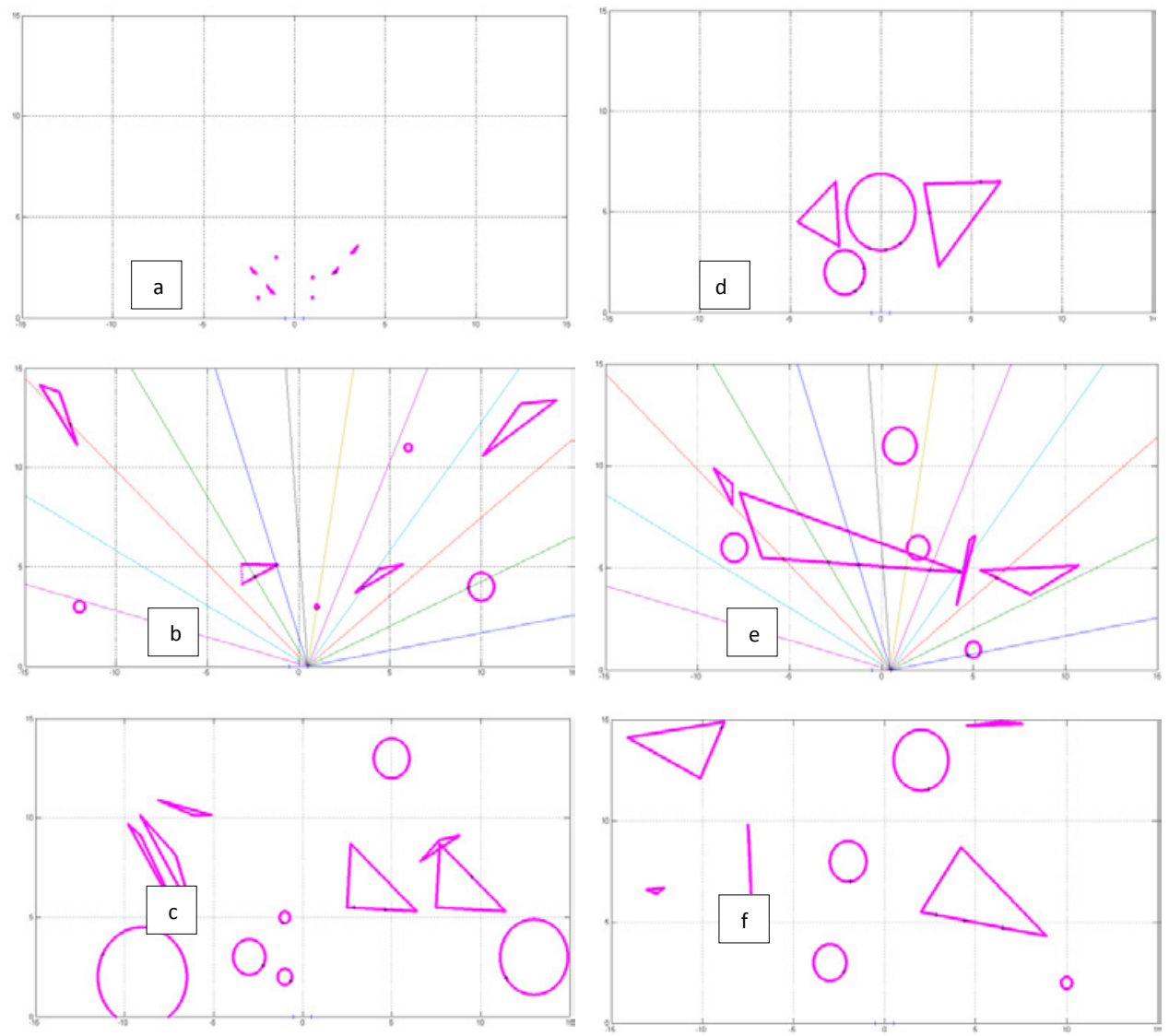

3. On Figure 5c uses12obstacles; scanning time was long in the most of angles.

4. On Figure 5e have a triangle with a wall behavior, in this phenomenon all the angles take more time versus others frames

In simulation data were enough to apply statistical methods (Simulation Graphic Shows: Detected Point Versus Opening Angles) for 101 experiments (Figure 6). The Figure 6 shows values of optimized scanning angles using: small angular increment of scanning step in simulation; the quantity of obstacles in scene 1-13 and laser length was $30 \mathrm{~m}$.

Every scene was analyzed using parameter $Z$ which called advance (The Joint Quantity of All Detected Points on Surfaces of All Obstacles inside the Scene) that was calculated:

$$
Z \equiv P / t
$$

Where $P$ is number of points detected on obstacles, $t$ is the scanning time in seconds.

Figure 7 represent simplified by maximum and minimum method, where $Z$ parameter is under hypothesis "Find three optimum aperture angles based on the data obtained from 101 scenes using Method connection means and the detected points vs. time", which show us the optimum angles in Figure 8.

In all possible values of scanning step there are three values of angle where the scanning velocity grow continuously but the quantity of

Figure 5: Frames from simulation in MATLAB. 
Citation: Garcia-Cruz XM, Sergiyenko OY, Tyrsa V, Rivas-Lopez M, Hernandez-Balbuena D, et al. (2013) Fast Obstacle Detection by Variable Step of 3D Laser Scanning for Robot Navigation on Unknown Planet. J Appl Mech Eng 2: 124. doi:10.4172/2168-9873.1000124

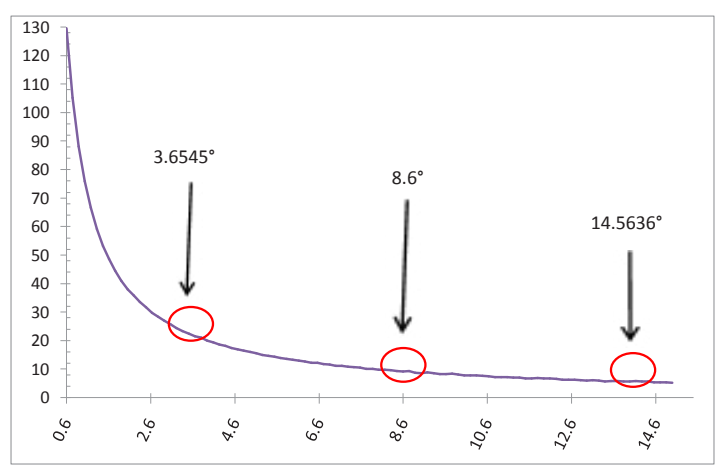

Figure 6: Detected points vs. opening angles from 101 simulation scenes [23].

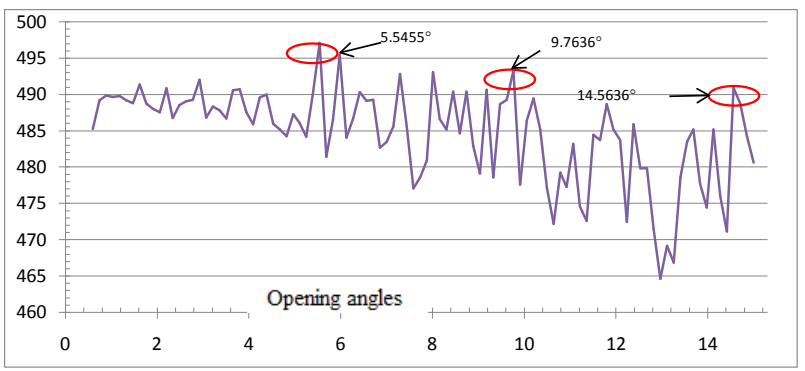

Figure 7: Z vs. Opening Angles.
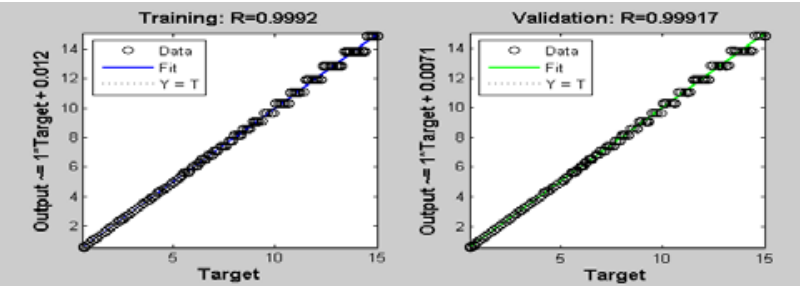

Test: R-0.99917
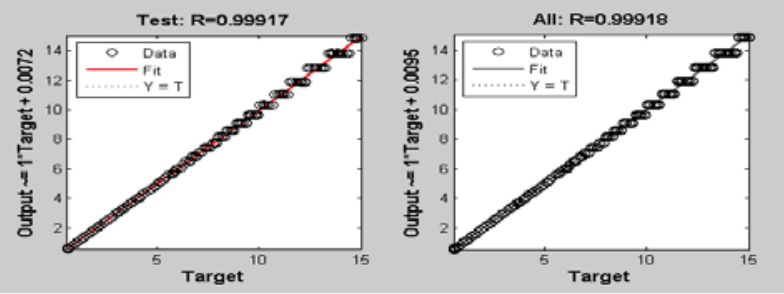

Figure 8: Training Regressions.

information about obstacle is changing with discrete level. Such angles are angles around $1.8-4^{\circ}$ (Blue Rows of Table 2 ), $8.2-8.7^{\circ}$ and $14-14.6^{\circ}$ (Green Row of Table 2). Such situation is very convenient for us in this particular task. Practically, it means the next.

The biggest angle (around $14^{\circ}$ ) permits us to scan the scene the fastest possible way, is the biggest angle for all provided 10100 simulations still not miss anyone of simulated obstacles. The angles of bigger value are already not detecting some obstacles. The next selected angle of $8.6^{\circ}$ is appropriated for fastest rough scanning, Table 1 shows analyzed data. Angle around $8.6^{\circ}$ seems to be the best candidates for optimal scanning, $8.6^{\circ}$ gives 4.42 times faster than $1.9091^{\circ}$ but still 1.72 times worse than $14.5636^{\circ}$. But at the same time is still 2.32 times faster than $3.65454^{\circ}$, but slower 1.11 times that $9.7636^{\circ}$ see Table 2 for comparing angles.

The last extreme experimentally obtained value of step angle around $1.8-4^{\circ}$ provides the slowest scanning; practically it is applicable only for precise 3D measurement on the edge of obstacle under highest interest for collision prevention in the further task of robot trajectory planning.

\section{Levenberg-Marquardt Method application for rectifying of optimal angles}

According to Rodriguez-Quinonez et al. [19] the most proper tool in this case is the Levenberg-Marquardt Algorithm, which is in the least squares curve fitting problem: given a set of " $m$ " empirical datum pairs of variables, $\left(x_{\mathrm{i}}, y_{\mathrm{i}}\right)$, optimize the parameters $\beta$ of the model curve $f(\mathrm{x}, \beta)$ so that the sum of the squares of the deviations becomes minimal $[3,21]$.

$$
S(\beta)=\sum_{i=1}^{m}\left[y_{i}-f\left(x_{i}, \beta\right)\right]^{2}
$$

Levenberg-Marquardt is an iterative procedure. Marquardt's contribution $[19,23]$ is to replace Levenberg equation by a "damped version",

$$
\left(\boldsymbol{J}^{T} \boldsymbol{J}+\lambda \boldsymbol{I}\right) \delta=\boldsymbol{J}^{T}[\boldsymbol{y}-\boldsymbol{f}(\beta)]
$$

Marquardt $[19,23]$ replace the identity matrix, I, with the diagonal of $\boldsymbol{J}^{T} \boldsymbol{J}$, resulting in the Levenberg-Marquardt algorithm:

$$
\left(\boldsymbol{J}^{T} \boldsymbol{J}+\lambda \operatorname{diag}\left(\boldsymbol{J}^{T} \boldsymbol{J}\right)\right) \delta=\boldsymbol{J}^{T}[\boldsymbol{y}-\boldsymbol{f}(\beta)]
$$

where $J$ is the Jacobian matrix $[19,22]$ whose $i^{\text {th }}$ row equals $J_{i}$, and where $f$ and $y$ are vectors with $i^{\text {th }}$ component $f\left(\mathrm{x}_{\mathrm{i}}, \beta\right)$ and $\mathrm{y}_{\mathrm{i}}$, respectively. This is a set of linear equations which can be solved for $\boldsymbol{\delta} . \boldsymbol{I}$ is the identity matrix, giving as the increment, $\boldsymbol{\delta}$, to the estimated parameter vector, $\boldsymbol{\beta}$. The (non-negative) damping factor, $\boldsymbol{\lambda}$, is adjusted at each iteration.

\begin{tabular}{|l|l|l|l|l|}
\hline $\begin{array}{l}\text { Opening } \\
\text { Angles }\end{array}$ & $\begin{array}{l}\text { Detected } \\
\text { Points' }\end{array}$ & $\begin{array}{l}\text { Scanning } \\
\text { time }(\mathbf{s e c})\end{array}$ & Resolution & $\begin{array}{l}\text { Conclusions comparing with } \\
\mathbf{8 . 6}^{\circ}\end{array}$ \\
\hline $1.9091^{\circ}$ & 41 & 0.084 & High & $\begin{array}{l}8.6^{\circ} \text { detecting } 32 \text { points less than } \\
1.9091^{\circ} \text { but was } 4.42 \text { times faster }\end{array}$ \\
\hline $3.6545^{\circ}$ & 21 & 0.044 & High & $\begin{array}{l}8.6^{\circ} \text { detecting } 12 \text { points less than } \\
3.65451^{\circ} \text { but was } 2.32 \text { times faster }\end{array}$ \\
\hline $5.5455^{\circ}$ & 14 & 0.029 & Medium & $\begin{array}{l}8.6^{\circ} \text { detecting } 5 \text { points less than } \\
5.5455^{\circ} \text { but was } 1.53 \text { times faster }\end{array}$ \\
\hline $9.7636^{\circ}$ & 8 & 0.017 & Low & $\begin{array}{l}8.6^{\circ} \text { detecting } 1 \text { point more than } \\
9.7636^{\circ} \text { but was } 1.11 \text { times more } \\
\text { slow }\end{array}$ \\
\hline $14.5636^{\circ}$ & 5 & 0.019 & Low & $\begin{array}{l}8.6^{\circ} \text { detecting } 4 \text { points more than } \\
14.5636^{\circ} \text { but was } 1.72 \text { times } \\
\text { more slow }\end{array}$ \\
\hline & & & &
\end{tabular}

Table 1: Comparison with scanning step angle of $8.6^{\circ}$.

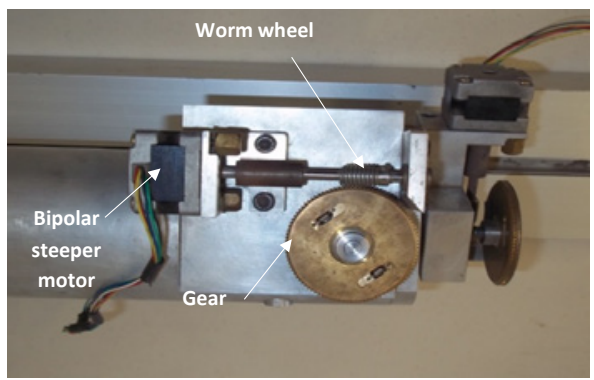

Figure 9: Laser positioning system of TVS [21]. 
Citation: Garcia-Cruz XM, Sergiyenko OY, Tyrsa V, Rivas-Lopez M, Hernandez-Balbuena D, et al. (2013) Fast Obstacle Detection by Variable Step of 3D Laser Scanning for Robot Navigation on Unknown Planet. J Appl Mech Eng 2: 124. doi:10.4172/2168-9873.1000124

Page 5 of 6

The LMA has been performed on system results. All information from simulation, was concentrated it in a matrix of [ $3 \times 10100]$, the data was used for preparing the Neural Network (NN) with of the LMA, using the next percentages: the training was $50 / 100$, validation $25 / 100$ (these are used to measure network generalization), testing 25/100 (when generalization stops improving). (NN) regression algorithm is effective for next angle prediction; it can verify which predicted values adjust to real values with $2 \%$ error (see Figure 8). Network is adjusted by error, and trained to acquire the constants of the scene components, based in the training it will decide which angle is the best for the next scanning.

\section{Gear box or MET}

MET is aimed to change adequately and in shortest time the step angle. Actually system $[14,20]$ has an anti-backlash gear 48 pitch $0.104^{*}$ face with $20^{\circ}$ pressure angle, 96 teeth, P.D 2.0000, gears 303 stainless steel, 2024-T4 Aluminum, hubs 303 stainless steel, the worm wheel has 48 pitch $20^{\circ}$ pressure angle, right hand double thread, the material it makes bronze ASTM B21 alloy 464, 12 VDC bipolar stepper motor with step angle $1.8^{\circ}$, holding torque $2100 \mathrm{~g}-\mathrm{cm}, 200$ steps per revolution $[14,20]$. The current design of laser positioning system of TVS is represented in Figure 9.

We were proposed an ideal electro-mechanical system [20] with variable opening angle as a Dual Clutch Transmission (DCT) [24-27]. However, as the present advanced research shows in a difference to [20] that optimal scanning angles are not so fixed, but the DCT permits due to its design only fixed transmission ratio, so we must to apply another principle than in [20]. The design needs especially three angles for three

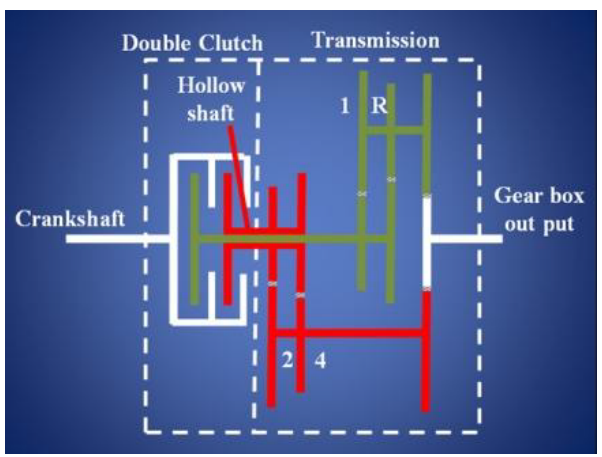

Figure 10: DCT proposed [21].

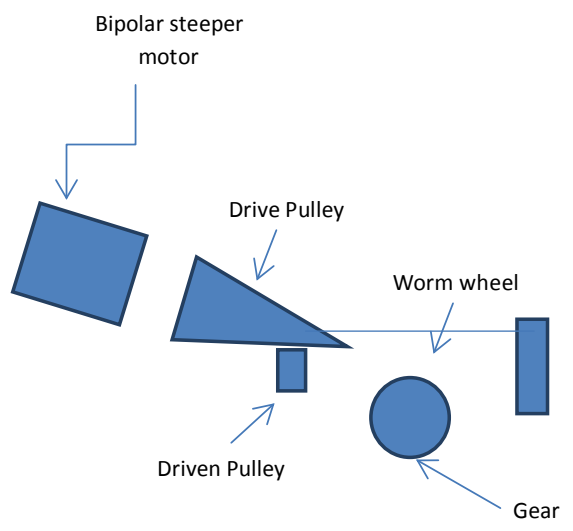

Figure 11: MET proposed [32]

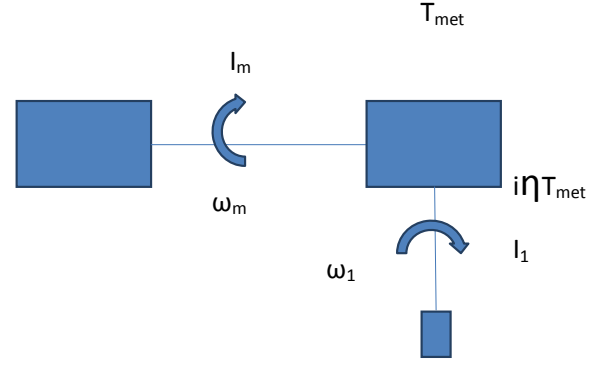

Figure 12: Simplified model of MET proposed [32].

\begin{tabular}{|l|l|l|l|l|l|l|}
\hline & $\begin{array}{l}\text { Optimize } \\
\text { time }\end{array}$ & $\begin{array}{l}\text { Using } \\
\text { energy }\end{array}$ & $\begin{array}{l}\text { Mechanical } \\
\text { maintenance }\end{array}$ & Weight & $\begin{array}{l}\text { Jumps } \\
\text { during } \\
\text { shifts. }\end{array}$ & Friction \\
\hline CVT & More & Less & Less & Less & Lees & $\begin{array}{l}\text { Influence of contact- } \\
\text { zone between chain } \\
\text { links on the torque } \\
\text { capacity and dynamic } \\
\text { performance [29] }\end{array}$ \\
\hline DCT & Less & More & More & More & More & $\begin{array}{l}\text { To avoid friction in this } \\
\text { type of transmission it } \\
\text { need hydraulic pres- } \\
\text { sure [27] }\end{array}$ \\
\hline
\end{tabular}

Table 2: Comparison CVT vs. DCT [21]

transmissions ratios supposedly to be that angles simulation brings us. For proposed DCT introduced on Figure 12 [20], the available space for TVS MET is around $0.115 \mathrm{~m}$ width and $0.03 \mathrm{~m}$ high; this space is not enough for proposed design on, for maintenance this kind proposed of transmission need lubricant for decrease friction, DCT need vary velocity in three rigorously fixed speed changes and reverse, see Figure 10 .

Analyzing Continuously Variable Transmission (CVT) vs. DCT (refer Table 2), for TVS application CVT is the better option, CVT is better in optimized time, minor energy use, low maintenance, less weight, and it avoids jumps during shift. For reduced friction CVT is better than DCT. Below will be explained DCT design for actual TVS.

Continue Variable Transmission uses two slots or pulleys that fit their axial widths simultaneously in opposite directions to change the transmission ratio This design has more than a century of use in the automobiles; snow blowers and lawn mowers [21,28], and this long experience in different applications with distinct conditions of use shows that CVT has an advantage: it is strongly reliable and low dependent on surrounding constrains. It is a good property to novel design with unknown practical restrictions. In Table 2 we analyzed conditions and parameters for CVT versus DCT [21].

Based on our data, previous design in research [20], we can decide the next. CVT has various sub-designs. We choose principle of CVT operation mostly matched to our case, for still more economy solution we propose the design without one cone and clutch. The design will put on novel MET type "Cone mesh CVT", in this design use one cone (which be called Drive Pulley) and one shaft (which be call Driven Pulley), for reference see Figure 11 [21].

MET proposed components and give it a representation, in Figure 12 can see simplified model of proposed MET.

We can obtain the dynamic equations of MET from Figure 12 [29]:

$I_{m} \omega_{m}+B_{m} \omega_{m}=T_{m}$ 
Citation: Garcia-Cruz XM, Sergiyenko OY, Tyrsa V, Rivas-Lopez M, Hernandez-Balbuena D, et al. (2013) Fast Obstacle Detection by Variable Step of 3D Laser Scanning for Robot Navigation on Unknown Planet. J Appl Mech Eng 2: 124. doi:10.4172/2168-9873.1000124

$$
I_{1} \omega_{1}+B_{1} \omega_{1}=i \eta T_{m e t}-T_{1}
$$

Where $I_{m}$ is the equivalent rotary inertia of motor, $I_{1}$ is the equivalent rotary inertia of the active pulley of MET, $\omega_{\mathrm{m}}$ is the angular velocity of motor, $\omega_{1}$ is the angular velocity of the active pulley of MET, $B_{m}, B_{1}$, represent the equivalent damping coefficient of each axis respectively; $\mathrm{i}$ is the speed ratio of MET and $\eta$ is the transmission efficiency. $T_{m}$ is the output torque of motor; $T_{1}$ is the output torque of laser ray drive (equivalent torque of all units sum of laser positioning system).

$$
T_{1}=F_{1} r=r\left[G+\left(m+\frac{\sum I_{2}}{r^{2}}+\frac{I_{f} i^{2} \eta}{r^{2}}\right)\right]
$$

In this equation, $r$ is the radius of laser ray drive; $G$ is the weight of all units sum of MET; $I_{f}$ is the rotary inertia of laser ray drive; $m$ is the mass of rotary parts [29].

According to our design presented on [20,21], the MET must to provide the scanning step angle value changes as soon as possible, and as evident from equation 15 and 16 there are no too much options to provide it. The majority of parameters in equations 15 and 16 are physical constants, even $\omega_{\mathrm{m}}$ is the constant in our offer because of the simplest and reliable design of TVS, and so, only $\omega_{1}$ is the unique candidate under consideration for its variable control function programming. In our opinion, this is another small advantage of our proposition: the simplest controls always are more robust. In fact, we propose to program the changes by system processor of the position of active pulley (see Figure 12) to satisfy the condition that the number value of ratio of controlled $\omega_{1}$ and constant $\omega_{\mathrm{m}}$ will deal exactly value of one angle of $3.65^{\circ}, 8.6^{\circ}$ and $14.6^{\circ}$, as necessary at the moment to provide scan[21].

\section{Conclusion}

This paper can offers the solution capable to increase velocity of obstacle detection to mobile robot navigation. Computational experiments in the present paper are obtained values of three particular angles which permit among others the minimal losses of information about scanned objects. That application permits accelerate the process of automatic search of the obstacles within the scene under interest, with posterior precise measurements only those edges of obstacles mostly close to future trajectory of the robot. Multivariable simulation of scenes in this software warranties the high reliability of such optimized scanning angles and the enhanced resolution of fast scanning.

Comparing two kinds of micro transmission design, dual transmission clutch and the conic, in a difference to our previous solution in [20]. Clearly shown by various simulation results that transmission conical more of a practice limits is best matched our practical application requirements.

\section{References}

1. Zhang Z, Huang S (2012) 3D Surface Shape Measurement based on Fringe Projection Techniques. J Appl Mech Eng.

2. Barone S, Paoli A, Razionale AV (2012) Shape measurement by a multi-view methodology based on the remote tracking of a 3D optical scanner. Optics and Lasers in Engineering 50: 380-390.

3. Abba A, Manenti A, Suardi A, Geraci A, Ripamontii G (2009) Non-linear leastsquares in fpga devices for digital spectroscopy. Nuclear Science Symposium Conference Record, Orlando, USA.

4. MacFarlane DL, Kim WY, Nilvi JS, Nuth T, Tan V (1992) Novel two-dimensional laser scanning technique. Optics and Lasers in Engineering 17: 1-8.
5. Basu A, Licardie S (1993) Modeling fish-eye lenses. Intelligent Robots and Systems 3: 1822-1828.

6. Zhu Z (1997) On Environment Modeling for Visual Navigation. PhD Tsinghua University, Beijing, China.

7. Zhu Z, Xu G, Lin X (1999) Panoramic EPI generation and analysis of video from moving plataform with vibration. IEEE Computer Society Conference on Computer Vision and Pattern Recognition.

8. Zhu, Zhigang (1993) Omnidirectional stereo vision. Proceedings of the workshop on Ominidirectional Vision, Buda Basu, Anup, and Sergio Licardie.

9. Sergiyenko OYu (2010) Optoelectronic System for Mobile Robot Navigation. Optoelectronics, Instrumentation and Data Processing 46: 414-428.

10. Fu G, Menciassi A, Dario P (2012) Development of a low-cost active 3D triangulation laser scanner for indoor navigation of miniature mobile robots. Robotics and Autonomous Systems 60: 1317-1326.

11. Sergiyenko O, Vera Tysra, Luis C, Julio C, Hernandez W, et al. (2011) Electromechanical 3D optoelectronic scanners: resolution constraints and possible ways of its improvement. IN-TECH, Vienna, Austria.

12. Xiong Zhihui, Chen Wang, Zhang Maojun (2008) Catadioptric Omni-directiona Stereo Vision and Its Applications in Moving Objects Detection. Computer Vision 493-518.

13. Básaca LC, Rodríguez J, Sergiyenko OY, Tyrsa VV, Hernandez W, et al. (2010) 3D Laser Scanning Vision System for Autonomous Robot Navigation. ISIE 2010, Bari, Italy.

14. Basaca LC, Rodríguez J, Sergiyenko OY, Tyrsa VV, Hernandez W, et al. (2010) Resolution improvement of dynamic triangulation method for $3 \mathrm{D}$ vision system in robot navigation task. IECON 2010, Glendale, AZ, USA.

15. Lopez MR, Sergiyenko O, Tyrsa V (2008) Machine vision: approaches and limitations. Computer vision.

16. Sergiyenko O, Tyrsa V, Hernandez B, Rivas Lopez, Crez D, et al. (2008) Precise Optical Scanning for practical multiapplications, Florida, USA.

17. Rodriguez JC, Sergiyenko OY, Tyrsa VV, Basaca LC, Hipolito JIN (2011) Continuous monitoring of rehabilitation patients with scoliosis using automatic laser scanning. Pan American Health Care Exchanges (PAHCE, 2011), Rio de Janeiro, Brazil.

18. Rivas M, Sergiyenko O, Aguirre M, Devia L, Tyrsa V, et al. (2008) Spatial data acquisition by laser scanning for robot or SHM task. ISIE 2008, Cambridge, UK.

19. Rodriguez-Quinonez JC, Sergiyenko O, Gonzalez-Navarro FF, BasacaPreciado L, Tyrsa V (2013) Surface recognition improvement in 3D medical laser scanner using Levenberg-Marquardt method. Signal Processing 93: 378386.

20. Garcia-Cruz XM, et al (2011) Optimization of Laser TVS for robot navigation using combined scanning with variable step. ROC \& C-2011, Acapulco, Guerrero, Mexico.

21. X.M. Garcia-Cruz (2013) Optimization of 3D laser scanning speed by use of combined variable step. Optics Laser Engineering, Elsevier.

22. Wilamowski BM, Yu H (2010) Improved computation for Levenberg-Marquardt training. IEEE Transactions on Neural Networks 21: 930-937.

23. Marquardt D (1963) An algorithm for least-squares estimation of nonlinear parameters. J Soc Indust Appl Math 11: 431-441.

24. Kulkarni M, Shim T, Zhang $Y$ (2007) Shift dynamics and control of dual-clutch transmissions. Mechanism and Machine Theory 42: 168-182.

25. Huai-Ching Chien, Ching-Huan Tseng (2004) An automatic transmission for bicycles: a simulation. International Journal of Industrial Ergonomics 33: 123132.

26. Galvagno E, Velardocchia M, Vigliani A (2011) Dynamic and kinematic mode of a dual clutch transmission. Mechanism and Machine Theory 46: 794-805.

27. Norton RL (2004) Design of machinery: an introduction to the synthesis and analysis of mechanisms and machines. McGraw-Hill Higher Education, New York, USA.

28. Meilan Z, Wen J, wang X, Zhou Y (2008) Modeling and performance simulation of transmission system for car equipped with metal pushing V-belt type CVT. IEEE Vehicle Power and Propulsion Conference, Harbin, China.

29. Srivastava N, Imtiaz Haque (2008) Clearance and friction-induced dynamics of chain CVT drives. Multibody System Dynamics 19: 255-280. 